\title{
Personas mayores, participación y cambio social antes y después del COVID-19
}

\author{
Elvira Medina Ruiz \\ Universidad de Murcia
}

\begin{abstract}
Resumen
La participación social está estrechamente ligada al bienestar y un envejecimiento saludable generando beneficios físicos y psicológicos. Sin embargo, las personas mayores tienen tendencia a bajar los niveles de participación e interacción social tras la jubilación, aunque otros muchos continúan militando y participando en entidades y movimientos sociales.

La pandemia provocada por la COVID-19 ha tenido un fuerte impacto a nivel social y sanitario siendo los principales perjudicados las personas mayores, no solo por las elevadas tasas de mortalidad sino porque las medidas de distanciamiento social impuestas para su protección, han dado lugar a un agravamiento del aislamiento social y el sentimiento de soledad de nuestros mayores. Es urgente generar espacios seguros para aumentar los niveles de participación social.
\end{abstract}

Palabras clave

Personas mayores; participación social; envejecimiento; COVID-19; aislamiento

Códigos JEL: L31, D63, D71, I31

\author{
OLDER PEOPLE, PARTICIPATION AND SOCIAL CHANGE \\ BEFORE AND AFTER COVID-19
}

\section{Abstract}

Social participation is closely linked to well-being and healthy aging, generating physical and psychological benefits. However, older people tend to lower levels of participation and social interaction after retirement although many others continue to militate and participate in non-profit organisations and social movements.

The pandemic caused by COVID-19 has had a strong impact at the social and health level, with the elderly being the main victims, not only due to the high mortality rates but because the social distancing measures imposed for their protection have led to a worsening of social isolation and the feeling of loneliness of our elders. It is urgent to create safe spaces to increase levels of social participation.

Keywords

Older people; social participation; ageing; COVID-19; isolation

JEL codes: L31, D63, D71, I31

Fecha de recepción del original: 18 de junio de 2021; versión definitiva: 30 de julio de 2021.

Elvira Medina Ruiz, Facultad de Trabajo Social, Campus Universitario de Espinardo, Universidad de Murcia. 30100 Murcia. Tel.: +34 868888102; E-mail: emedina@um.es; ORCID ID: https://orcid.org/0000-0003-4937-3345. 


\section{Personas mayores, participación y cambio social antes y después del COVID-19}

Elvira Medina Ruiz

Universidad de Murcia

1. Introducción

La población de personas mayores es muy numerosa en todos los países desarrollados. Los importantes avances en las ciencias de la salud, el desarrollo de nuevas técnicas geriátricas y gerontológicas, unidos a mejoras del nivel de vida han dado lugar a una disminución de la mortalidad y aun aumento de la esperanza de vida.

Las personas mayores son un importante recurso en expansión que ofrece multitud de habilidades, tiempo y experiencia (Medina, 2005). Su contribución a la sociedad es un tema que ha ido ganando importancia en los últimos años, pero todavía suscita incertidumbres e incluso recelos.

La jubilación a menudo funciona como punto de inflexión en el que se puede producir una importante pérdida de poder adquisitivo, una reducción de las relaciones sociales, que antes podían estar vinculadas al ámbito laboral y con ello, una pérdida de oportunidades de participación.

Además, tras la jubilación, se produce también una pérdida de valor social de los individuos. En la sociedad tradicional los conocimientos y habilidades acumulados por las personas mayores suponían un activo imprescindible para el desarrollo de las sociedades, pero en la sociedad posindustrial, que experimenta un desarrollo y cambio continuo, sus experiencias y habilidades quedan obsoletos en poco tiempo, perdiendo la vigencia de su función social y disminuyendo su estatus o prestigio en la comunidad (Bjørkøy, 2017, citado por Amezcua y Alberich, 2020).

Afortunadamente, no todos los mayores se encuentran en la misma situación. Pese a que a partir de los 65 años la mayoría de personas dejan de trabajar, muchas permanecen activas contribuyendo al tejido social o participando activamente en muchas esferas de la vida pública y privada. Las personas mayores son un importante activo para las entidades y movimientos sociales, ya que son capaces de aportar tiempo y conocimiento en grandes cantidades, llegando incluso a fundar y gestionar importantes movimientos como fueron los laioflautas en 2012 o la Marea de Pensionistas unos años más tarde, con una importante capacidad de incidencia política.

Sin embargo, según datos de EU-SILC Survey de Eurostat para 2015, los niveles de participación de nuestros mayores en España siguen por debajo de la media europea que se sitúa en $29,2 \%$ y alejada de muchos países como Alemania con más de un $54 \%$. El fomento y la visibilidad de la participación social, por tanto, sigue siendo uno de los retos pendientes de la sociedad española pese a que hace ya más de veinte años que se relacionó directamente bienestar y envejecimiento activo y saludable con el mantenimiento de la participación social.

¿Por qué entonces no se potencia más desde las administraciones públicas o las entidades privadas? Quizás la respuesta esté precisamente en la importante incidencia política y el poder de movilización que pueden tener las personas mayores mediante acciones coordinadas y organizadas.

Pero si ya nos preocupaba la baja participación de nuestros mayores, esta, se ha visto agravada con la crisis sanitaria y social provocada por el COVID-19. A raíz de la pandemia corremos el riesgo de sufrir un retroceso en la participación y la presencia de las personas mayores en los espacios públicos por miedo al contagio, lo que puede afectar a incrementar su aislamiento social y sentimiento de soledad empeorando así su salud física y mental.

El presente trabajo aborda el fenómeno de la participación social de las personas mayores, y expone los riesgos ante las duras cifras que nos ha dejado la pandemia en 2020. Se hace urgente repensar la manera de recuperar de manera segura, la dimensión pública de los mayores para no retroceder años, donde los mayores solo tenían un papel destacable en la esfera privada y familiar.

2. La importancia de la demografía. El perfil de las personas mayores en España

España es uno de los países más envejecidos de la Unión Europea. Según los últimos datos del Instituto Nacional Estadística, en nuestro país hay más de 9 nueve millones de personas mayores de 65 años, lo que supone un $19,4 \%$ del total de la población (INE, 2021). Que el continente europeo envejezca a un ritmo alto tampoco es algo nuevo, y son muchos los países que vienen tomando medidas para afrontar esta estructura demográfica que, sin duda, pasa por medidas de tipo no solo económicas sino sociales, políticas y culturales. La revolución cada vez me- 
nos silenciosa del envejecimiento debe ser una prioridad política más si cabe a raíz de la crisis sanitaria.

En cuanto al perfil de nuestros mayores, el número de mujeres de 65 y más es de 5.067 .179 frente a los 3.838.559 varones. O lo que es lo mismo, el $57 \%$ son mujeres (INE, 2018a). Se explica porque la esperanza de vida de las mujeres no solo es mayor, sino que ha sido subiendo considerablemente en las últimas décadas. En 1900, la esperanza de vida de las mujeres era de 37,7 y la de los hombres, 33,9. En 2017, la esperanza de vida de las mujeres era de 85,7 y la de los hombres, 80,4 (INE, 2017). Pero no solo eso, la esperanza de vida al nacimiento en España es de 83,1 años. La esperanza de vida al nacer en España es la más elevada de la Unión Europea, seguida de Italia, Francia, Luxemburgo y Suecia, con esperanzas de vida por encima de los 82 años (INE, 2018a).

Según predicciones del Instituto Nacional de Estadística (INE), en 2033 el 25,2 \% de la población superará los 65 años. Si se mantienen constantes los ritmos actuales de reducción de la incidencia de la mortalidad por cada grupo de edad, en 2033 la esperanza de vida al nacer será de 82,9 años para los varones y de 87,7 para las mujeres (INE, 2018a). Es cierto que estas cifras son en contexto de prepandemia. Todavía es pronto para afirmar si ha habido o va a haber alguna modificación relevante tras la crisis del COVID-19.

La formación y el nivel de estudios es algo que también ha interesado a los investigadores, sobre todo a la hora de relacionarlo con los niveles de participación social y voluntariado (Medina, 2015). En España, cinco de cada diez personas mayores tiene estudios primarios y un $7 \%$ es analfabeta. No obstante, la brecha de género sigue siendo acusada. Entre los hombres, el $50 \%$ tiene estudios primarios, el $29 \%$, secundarios, el $18 \%$ ha cursado estudios superiores y solo un $3 \%$ es analfabeto. Por su parte, entre las mujeres, el $58 \%$ tiene estudios primarios, el $27 \%$, secundarios, no llegan al $10 \%$ las mujeres mayores con estudios superiores y el analfabetismo alcanza al 6 \% (INE, 2018a).

Además, cerca del $1 \%$ de los mayores de 65 años sigue manteniendo alguna actividad laboral. Las más frecuentes son: las relacionadas con el comercio, las del sector primario, las actividades científicas, profesionales y técnicas, así como las sanitarias y de servicios sociales (INE, 2018b).

3. La participación social de las personas mayores: vehículo para el cambio social

\subsection{El papel de los mayores en la sociedad}

A la hora de describir los avances y cambios de las sociedades industriales actuales, la familia siempre ha sido un elemento de referencia para explicar los procesos de cambio. La familia es uno de los núcleos básicos de la so- ciedad y constituye una realidad plural, dinámica y compleja en constante evolución (Vázquez de Prada, 2005).

Por ejemplo, el aumento de la esperanza de vida, ha afectado a la estructura familiar permitiendo que varias generaciones convivan mucho más tiempo y al envejecer en mejores condiciones tanto físicas como psicológicas y sociales, también ha permitido a las personas mayores a ejercer roles más importantes en el seno de la familia (Posner, 1995).

Hoy en día, son muchos las personas mayores que cuidan de sus nietos para poder ayudar a sus hijos. Esto además de haber ido en aumento en los últimos años, fue un importante activo tras la recesión de 2008 , en la que muchos mayores se convirtieron en los sustentadores principales de muchos hogares aportando su tiempo y dinero para el cuidado y mantenimiento de sus hijos y nietos (Educo, 2015). Pero a menudo, lejos de ser una carga, esta tarea reporta una serie de sentimientos positivos a los mayores, como alegría, satisfacción y sentimientos de utilidad principalmente y lo hacen por el placer de dar sin esperar nada a cambio (Family Matters Institute, 2009) pero a la vez, contribuyen de manera indirecta al bienestar de los nietos, a través del ofrecimiento de apoyo emocional (Morgado y Román, 2011).

En algunos contextos, la prolongación de la vida humana además de ser un detonante de cambio está pasando a ser percibida más que como una conquista del género humano como un problema social y la recién crisis sanitaria provocada por el COVID-19 así lo ha demostrado. Esta situación aparentemente paradójica sé aplica por la necesidad de un Estado Social de realizar traspasos de renta que son cada vez más importantes en los colectivos que experimentan mayores privaciones. En el caso de las personas mayores, los gastos sociales se incrementan no solo por el paso de la vida económicamente activa a la pasiva, sino también porque la edad avanzada conlleva en general un aumento de los problemas de salud y de la necesidad de asistencia.

Al analizar la situación social de las personas mayores en España se debe tener en cuenta cada vez más las diferencias por edad, nivel económico, situación social, etc. Existe una idea estereotipada general que acostumbre a contemplar a las personas mayores como un grupo homogéneo. Su heterogeneidad, por el contrario, lleva a estudiar dicha situación considerando las diferencias por género, nivel de salud, subgrupos de edad y clase social, lo que conlleva estilos de vida diferentes que conducen a las personas a vivir experiencias de envejecimiento distintas. Ya sabemos a la par que envejece la sociedad, envejece también el grupo de las personas mayores con cada vez más presencia de personas mayores de ochenta años (INE, 2021).

Otros temas de gran impacto social se han centrado en el estudio de la jubilación y la prejubilación desde variados posicionamientos, económicos, sociales, de servicios asistenciales y psicológicos, entre los más comunes. A lo largo de los años también ha sido resaltable el abordaje del fenómeno de la dependencia y la necesidad de atención antes y después de la promulgación de la Ley de Dependencia en 2006. Sin embargo, este tema está llamado a seguir siendo investigado y debatido pues la crisis del 
coronavirus ha puesto de nuevo sobre la mesa la multitud de fallas que tiene nuestro Sistema de Atención a la Dependencia, sobre todo en lo concerniente a los cuidados de larga duración o los centros residenciales.

Junto al estudio siempre importante de los cambios demográficos deben también tener en cuenta planteamientos de análisis que no olviden los sistemas sanitarios, sociales y educativos. Pero a estos planteamientos hay que sumarle el gran olvidado en el abordaje del envejecimiento, la participación social.

Quizás a nuestro juicio esta última resulta especialmente urgente puesto la pandemia provocada por el COVID puede hacer retroceder algunas de las metas y victorias alcanzadas en materia de participación social y voluntariado de los últimos años. Se trata de continuar elevando al máximo la capacidad de las personas mayores para vivir en sociedad, permitiéndoles llevar una vida lo más independiente posible en su propio hogar y en su comunidad, como ciudadanos activos y útiles. No como simples sujetos pasivos receptores de la atención de un sistema de servicios sociales o sanitario.

En los últimos años, desde organizaciones tanto públicas como privadas, se está insistiendo en la necesidad de potenciar la participación de las personas mayores. Como hemos visto en el epígrafe anterior, el peso demográfico de las personas mayores en los próximos años es innegable, pero esta tendencia debe ir acompañada de la presencia de los mayores en la vida pública, potenciando su participación social. La aparición del término "envejecimiento activo" es lo que mejor resume la tendencia que debemos seguir en los próximos años.

A finales de los años 90, la Organización Mundial de la Salud acuñó el término "envejecimiento activo" para expresar la idea de que el envejecimiento debe ser una experiencia positiva. Cumplir años debe ir acompañado de continuas oportunidades de salud, participación, aprendizaje y seguridad (IMSERSO, 2011).

La Organización Mundial de la Salud define el envejecimiento activo como "el proceso de optimización de oportunidades de salud, participación y seguridad con el objetivo de mejorar la calidad de vida a medida que las personas envejecen". Pese a que el término se acuñara en los 90 y en los últimos años se han hecho esfuerzos de para alcanzarlo, estos sobre todo han ido encaminados a mejorar la calidad de vida desde el punto de vista de salud, sin embargo, estamos lejos de tener un gran plan para el fomento de la participación social o la presencia de mayores en movimientos sociales o entidades de voluntariado.

El envejecimiento activo no es solo una expresión reciente o una moda. Tampoco es solo la idea básica que ya propugnaban los pioneros de la Teoría de la Actividad y el Envejecimiento Exitoso (Havighurst y Albretch, 1953; Havighurst, Neugarten y Tobin, 1963), es la expresión que refleja la realidad de cada vez más personas mayores (Agulló, Agulló y Rodríguez, 2002).

El envejecimiento activo permite a las personas mayores seguir desarrollando su potencial físico, social y mental. Numerosos estudios apuntan ya a un retraso de la discapacidad y la dependencia, aunque hay que ser cautos porque también muestran resultados contradictorios (Chirstensen, Doblhammer, Rau y Vaupel, 2009).
La entrada a la vejez, muchas veces marcada por la jubilación, empieza a reconocerse como un buen momento para introducir cambios en la vida, desarrollar nuevos proyectos, cuidar la salud y hacer ejercicio, en definitiva, hacer cosas que no se habían hecho durante la vida adulta (IMSERSO, 2011) pero esto también debe estar apoyado bajo el paraguas de las administraciones públicas.

Tengamos en cuenta que la actividad se convierte en un importante indicador de la calidad de vida global de los mayores (Agulló, et al., 2002). Autores como Triadó (2005) afirman que las personas mayores que realizan actividades de ocio muestran niveles más elevados de satisfacción vital, mayor energía, motivación y autoestima. Para Bowling y Grundy (2008), la participación social y las actividades de ocio de las personas mayores tienen relación directa con la autoestima y la satisfacción vital y también se asocian con menores riesgos de muerte. Para Fernández-Ballesteros (2003) las relaciones sociales también son una condición indispensable para tener un envejecimiento óptimo.

Naciones Unidas en su resolución 46/91 ya señalaba los principios en favor de las personas mayores para "dar más vida a los años que se han agregado a la vida".

Algunos de esos principios están directamente relacionados con práctica social y ya los señalaba Navarro Carrió (2000):

- La necesidad de permanecer integrados en la sociedad, participar activamente en la formulación y aplicación de las políticas que afecten directamente a su bienestar y poder compartir sus conocimientos y habilidades con las generaciones más jóvenes.

- Poder buscar y aprovechar oportunidades de prestar servicios a la comunidad y de trabajar como voluntarios en puestos apropiados a sus intereses y capacidades.

- Poder formar movimientos o asociaciones de personas de edad avanzadas.

Ya entonces Navarro Carrió mencionaba lo imprescindible de contar con los ciudadanos mayores y el movimiento asociativo para el diseño de las políticas sociales locales, al final y al cabo, la mayoría de las competencias en materia de servicios sociales y asistenciales están descentralizadas y corresponden a las Comunidades Autónomas y ayuntamientos.

Pero todavía hoy, una de las principales dificultades en el acercamiento al fenómeno de la participación de las personas mayores es que no existe suficiente información sobre ello (Medina, Marcos-Matás y Medina 2016).

\subsection{La participación social de las personas mayores}

Vega, Delgado y Martínez (2002) sostuvieron que la participación social es una herramienta que se debe impulsar debido a que la calidad de vida guarda una estrecha relación efectiva con ella, es beneficiosa en la esfera familiar y social, aumenta la autoestima de las personas y 
la valoración global que la persona hace sobre su vida es más positiva.

Si atendemos a Miranda et al. (2006), la actividad en la que participa una persona mayor es importante, pero más significante es para la persona que ejecuta la tarea, porque si se tratan de tareas que son de agrado hacia la persona, provoca que el envejecimiento se acepte y se lleve mejor, aumenta la alegría, el bienestar y la calidad de vida. Sin embargo, no aceptar bien los cambios y no participar de manera satisfactoria, provoca daño, un desgaste continuo.

Los autores Hernandis, Ruiz, Martí y Molina (2019) relacionan la participación social y la satisfacción vital, explicando que la participación social requiere un compromiso con la sociedad en todos los ámbitos. La correlación entre las personas y su ambiente genera efectos positivos en la salud física y psicológica, y prolonga los años de vida de las personas.

En 1991, la Asamblea de Naciones Unidas, en sus Principios a favor de las personas mayores subrayó la idea que "deberán poder buscar y aprovechar oportunidades de prestar servicio a la comunidad y de trabajar como voluntarios en puestos apropiados a sus intereses y capacidades". Han pasado muchos años desde la recomendación que hacía Naciones Unidas y es cierto que cada vez se nombra más a las personas mayores voluntarias, pero en el fondo poco se sabe sobre ellas. Hace años que se habla de una eclosión del movimiento asociativo de las personas mayores (véase en Kelly, 1993; Calero et al., 1996; Agulló et al., 2002; Medina, 2005; IMSERSO, 2008; Medina, 2015; Medina, Marcos-Matás y Medina, 2016), pero seguimos sin tener mucha información sobre esta importante tendencia.

En países como Australia, Estados Unidos o Canadá se llevan años dedicando esfuerzos especíicos para la atracción de las personas mayores al campo del voluntariado (IMSERSO, 2008). En Europa encontramos algunos ejemplos en Francia y Reino Unido (Hatto-Yeo, 2007). España no es muy diferente al resto de países europeos, siendo aún pocos los esfuerzos concretos que se realizan para potenciar el voluntariado de mayores. De hecho, según el Barómetro de UDP (2013) solo un 17,6 \% de las personas mayores declaran haber recibido en alguna ocasión una propuesta para hacer voluntariado en una ONG, asociación o entidad social.

Serrat Fernández (2016) diferencia dos tipos de participación, por un lado, la participación social como la unión de personas sin una causa o razón política y, por otro lado, la participación política, que hace referencia a los hechos encaminados a contribuir en el proceso de elegir entre distintas opciones políticas. Es en esta última donde se ubicarían los movimientos sociales y las mareas ciudadanas que han aumentado de peso en los últimos años y que tienen un considerable número de mayores en sus filas.

A través de la publicación elaborada por el IMSERSO en 2008 que abordaba en profundidad el fenómeno de la participación social de las personas mayores en España, podemos hablar de diferentes áreas temáticas:

Participación política referente al ámbito político; Participación laboral recoge la participación en el mercado de trabajo; Participación en el ámbito educativo tanto formadores como alumnos; Participación social restringido a la participación en asociaciones, movimientos sociales, acciones solidarias, conciencia ecológica y voluntariado; Participación y medios de comunicación referente a la participación de las personas mayores en los medios de comunicación como la prensa, la radio y la televisión; Participación en actividades de ocio y tiempo libre referente a la realización de actividades de ocio y tiempo libre (IMSERSO, 2008).

La importancia de la política en la vida de las personas mayores es relevante. Esto es opuesto al pensamiento de que las personas mayores no se preocupan por la política o que no tienen interés por ella. Además, con el aumento demográfico de los mayores, así como de su participación, pueden llegar a cambiar el resultado de las elecciones, pues según Riesco (2014:452) afirmó que "son precisamente las personas de 65 y más años las que manifiestan mayor interés por la política, aunque sea por una pequeña diferencia con la generación siguiente".

La participación de las personas mayores no es solo un instrumento de incidencia política, sino también un medio de inclusión social y mantenimiento de los vínculos con la comunidad que puede haberse visto afectada tras la pérdida de relaciones ocurrida tras la jubilación (Amezcua y Alberich, 2020).

Para Rubio (2004: 8), citado por Amezcua y Alberich (2020: 4), la movilización social es resultado de la interacción entre las condiciones estructurales y la acción. Desde la perspectiva de la "teoría de movilización de recursos", aparte de la identificación de objetivos comunes para la acción, es imprescindible analizar las circunstancias que favorecen que el descontento de las personas mayores se traduzca en movilización social y entre las que desatacan "los recursos, la organización y las oportunidades como medios que posibilitan la movilización y la consecución de objetivos".

Como ya hemos citado anteriormente, las personas mayores suponen un importante activo para la participación y también para el activismo político, pero para ello también se necesita un clima idóneo que favorezca el surgimiento, mantenimiento y alimentación de los movimientos sociales (McAdam, McCarthy y Zald, 1999).

En este sentido, varios autores piensan que el clima generado tras las movilizaciones en España y Europa tras el $15 \mathrm{M}$ generó un ambiente favorable. Pese a que el movimiento $15 \mathrm{M}$ se relacionó desde el principio con una población más joven, el clima de protesta y descontento producido a raíz de la gran crisis del 2008 y la política de recortes establecida por todos los gobiernos europeos, dio pie a varios movimientos de indignados como las mareas ciudadanas e incluso los laioflautas en Barcelona en 2011.

4. Efectos de la COVID-19 sobre la participación y vida social de los mayores

En enero de 2020, la Organización Mundial de la Salud (OMS) declaró el brote de la enfermedad por el nuevo co- 
ronavirus SARS-CoV-2 (COVID-19) como una emergencia de salud pública de alcance internacional, lo que acabaría convirtiéndose en la mayor pandemia y crisis sanitaria de las últimas décadas. Desde entonces, tanto la OMS como las autoridades sanitarias de todos los países colaboran para poder controlar y frenar sus efectos.

La crisis desatada por la pandemia también ha visibilizado las vulnerabilidades estructurales preexistentes $y$, a la vez, viene generando vulnerabilidades específicas emergentes de esta nueva situación. Esta situación está generando altos niveles de estrés individual y colectivo. Para personas en todo el mundo implica una situación trágica a causa las pérdidas que deben afrontar: pérdida de familiares y seres queridos, de la salud, de la vivienda, de bienes, o del empleo.

En España, la cifra de fallecidos a principios de junio de 2021 según el Ministerio de Sanidad era de 80.501 de un total de 3.733 .600 afectados.

Pero si en España se ha puesto en evidencia la fragilidad del sistema y las tremendas fallas que tenía, esto se ha notado todavía más en otras latitudes. Precisamente, la Organización Panamericana de Salud (OPS) decía que además de comprobar que la COVID-19 se ha cebado con la población mayor, la propia idiosincrasia de la Región requiere un cambio de modelo en el tratamiento y gestión de las necesidades de los adultos mayores.

Según la OPS, la pandemia ha:

- resaltado las necesidades y las vulnerabilidades que tienen las personas mayores con respecto a su derecho a la salud;

- evidenciado las tasas de mortalidad más altas en personas mayores con comorbilidades y con deterioro funcional; y

- expuesto la fragilidad de los sistemas de salud para apoyar a los adultos mayores y considerar sus necesidades únicas, entre otras.

Como muchos autores vienen manifestando los últimos meses, la pandemia puede ser una oportunidad para introducir importantes cambios en materia de política social y sanitaria en el tratamiento de las personas mayores.

La pandemia ha alterado muchas de las cosas de la vida cotidiana como la manera de vivir y disfrutar el ocio, el deporte, el trabajo o la propia forma de relacionarnos. Ha cambiado incluso nuestras percepciones y comportamientos sobre los demás, sobre otros grupos y el mundo en general. En algunos casos corriendo el riesgo de derivar en conductas discriminatorias como la aporofobia o el odio al inmigrante. De entre esas conductas discriminatorias, algunas investigaciones alertan ya de un aumento del edadismo (Ayalan et al., 2020).

El reduccionismo a la categoría de mayores e incluso "viejos", que se ha podido observar en medios de comunicación y discursos políticos en todo el mundo a la hora de abordar la crisis sanitaria, olvidando como dicen Fingerman y Trevino (2020) su heterogeneidad en la genética, historial de salud y enfermedades, historia de vida, modos de envejecer, y obviando que son agentes de cambio y ciudadanos de pleno derecho ha contribuido al incremento del edadismo.
Estos discursos edadistas y negativos, además de devaluar la propia imagen de las personas mayores contribuyen al sentimiento de inutilidad de las personas mayores, en el sentido de que suponen una carga y no un valor añadido para un país. Esto sumado a las restricciones que han tenido que asumir las personas mayores durante la pandemia, dada su extrema vulnerabilidad, ha podido afectar negativamente a su salud, aumentado las situaciones de aislamiento y soledad (Brook y Jackson, 2020).

Ya sabemos que la soledad es un factor de riesgo para el deterioro de la salud y el bienestar de todas las personas, pero los mayores son más vulnerables y propensos a sufrirla. Las relaciones sociales tienden a descender con la edad debido entre otras cosas, al desarrollo de afecciones a largo plazo, el deterioro de la salud, la muerte de parejas y amigos, por lo que las personas mayores pueden tener menos relaciones más cercanas y es más probable que vivan solas (Victor y Bowling, 2012).

Las barreras físicas y ambientales también impactan en el aislamiento social y la soledad de las personas mayores, como el sentimiento de inseguridad en sus vecindarios o durante la pandemia, el miedo al contagio. Este hecho también se puede ver agravado por una falta de políticas y medidas para favorecer y apoyar la socialización de los mayores, lo que puede acabar derivando en aburrimiento, desgana e inactividad (Cohen-Mansfield, Hazan, Lerman y Shalom, 2016).

Quizás lo que más preocupe ahora a los investigadores son los sentimientos de inseguridad y el deterioro de la salud psicológica y emocional a raíz de la pandemia y el aislamiento. Muchas personas están viendo seriamente afectada su salud mental y los efectos, similares a los de un estrés postraumático, es posible que sean duraderos (Pinazo-Hernandis, 2020). La soledad de nuestros mayores ya era un problema antes de la pandemia. En 2017, se calculaba que en España había casi dos millones de personas mayores viviendo solas. Y de esos hogares, tres de cada cuatro eran habitados por mujeres (INE, 2017).

El aislamiento social y la soledad son conceptos relacionados pero distintos. El aislamiento social se refiere a la falta de contacto o separación física de la familia, amigos o redes sociales más amplias y la falta de participación en actividades sociales (Valtorta y Hanratty, 2012) y se debe a restricciones ambientales, más que a la capacidad de un individuo para crear. o mantener relaciones sociales (Tanskanen y Anttila, 2016).

La soledad es una emoción subjetiva compleja, experimentada como un sentimiento de ansiedad e insatisfacción asociado con una falta de conexión o nexo con los demás, y un déficit entre la calidad y cantidad real y deseada de compromiso social (Victor, Scambler, Bowling y Bond, 2005). El aislamiento social y la soledad están correlacionados, aunque débilmente (Steptoe, Shankar, Demakakos y Wardle, 2013); sin embargo, ambos términos es habitual que se confundan y se usen de manera indiscriminada.

Como ya es bien sabido, la soledad y el aislamiento social aumentan el riesgo de ansiedad, depresión, disfunción cognitiva, enfermedades cardíacas y mortalidad de las personas mayores (Barth, Schneider y Kanel, 2010; HoltLunstad, Smith y Layton, 2010; Santini et al., 2020; Shankar, McMunn, Demakakos, Hamer y Steptoe, 2017). De ahí la 
especial preocupación de muchos por la situación de confinamiento derivada de la pandemia.

Antes de la pandemia, algunos gobiernos, conscientes de esta situación, habían implementado programas para fomentar la participación y disminuir las situaciones de aislamiento social. En el Reino Unido, the Campaign to End Loneliness (2011) creó una red de organizaciones nacionales, regionales y locales para trabajar juntas para garantizar que la soledad de las personas mayores siga siendo una prioridad de salud pública. Algo similar había implementado ya Nueva Zelanda en 2001 (Ministry of Social Development, 2001).

Tampoco el aislamiento o el miedo al contacto y la consecuente reducción de contacto social haya sido infundado, no solo tras la declaración del estado de alarma sino porque la consigna ha sido proteger a nuestros mayores mediante el confinamiento y la reclusión dado que el COVID-19 se ha cebado con los mayores. Pese a todo, de cara a futuro, tengamos en cuenta que la soledad ya era un reto para las asociaciones y administraciones antes de la pandemia, que es posible que muchas situaciones se hayan agravado a consecuencia de ella y proseguirán tras la amenaza del COVID-19 y que lo que esta pandemia nos plantea en términos de relaciones sociales, especialmente de la importancia de los recursos personales para hacer frente al aislamiento y la soledad (Fundación La Caixa, 2020).

Lo que está claro es que se necesita una estrategia y un enfoque integral, organizado y coordinado entre Administraciones, organizaciones sociales y resto de sociedad civil para dar respuesta a las personas mayores y mitigar, lo antes posibles las posibles situaciones negativas derivadas del confinamiento, el aislamiento y la discriminación por edad.

\section{Conclusiones}

El cambio demográfico en España en las últimas décadas ha sido espectacular: el porcentaje de personas de más de 65 años era el 9,7\% en 1971 y se sitúa ya cerca del $20 \%$ en la actualidad, lo que supone su casi duplicación en 40 años. A la inversa, el porcentaje de jóvenes se ha reducido casi a la mitad, sobre todo por las bajas tasas de natalidad.

El envejecimiento de la población supone un reto para cualquier país que se ve en la necesidad de aumentar y mejorar recursos para dar respuesta a las necesidades de esta población en los ámbitos que configuran los pilares fundamentales de una sociedad moderna.

La jubilación conlleva mayoritariamente, una pérdida de poder adquisitivo, así como una disminución de las redes sociales, muchas asociadas a los ámbitos de trabajo y una importante reducción de las posibilidades de participación. A esto se le suma, que, tras la gran recesión de 2008, muchos jubilados y mayores tuvieron que ayudar y sostener económicamente a sus hijos y nietos (Educo, 2015). Lo que aumentó considerablemente el riesgo de exclusión social.
La participación es un elemento muy importante de la dinámica social (Del Barrio, 2007), además de ser considerado un determinante clave del envejecimiento saludable (UDP, 2013). Cada vez hay más personas mayores, y cada vez más participan y forman parte de la sociedad de una manera activa. Actualmente estar jubilado no quiere decir pasividad, sino solo jubilación del mundo laboral.

La vejez se debe entender y afrontar como una etapa optimista. Los mayores tienen mucho que aportar tanto al ámbito familiar, como a nivel comunitario, por ejemplo, participando en movimientos asociativos o siendo voluntarios. No debemos perder de vista que los mayores han tenido un peso muy importante en nuestra sociedad y corremos el riesgo de ser absorbidos por los valores de utilidad-eficacia, pudiendo estigmatizar a las personas mayores (Cáritas Española, 2009).

Pero el creciente peso demográfico también puede incidir en un creciente peso político y socioeconómico: el gran poder de voto que puede modificar considerablemente unos resultados electorales (Amezcua y Alberich, 2020) como puede ser el resultado de las movilizaciones de la Marea de Pensionistas.

Se necesita reconocer la contribución que las personas mayores aportan a España, y lo importantes que son en el presente y que seguirán siéndolo en el futuro, por sus conocimientos adquiridos a lo largo de toda su vida. Sostiene que se debe romper con los estereotipos negativos, considerando a las personas mayores como personas independientes, con capacidad para relacionarse con quienes conviven y con el lugar en el que viven.

Los mayores deben de disponer de un espacio para desarrollar plenamente su participación activa en la sociedad en los diferentes planos: social, económico, cultural, educativo, político, etc. Hay que desarrollar donde no lo haya, los cauces necesarios que conduzcan a conseguir esa aspiración, fomentando el asociacionismo, apoyando los movimientos que ya existen y haciendo posible el acceso a la toma de decisiones en las que se traten temas que les conciernan tanto directamente como no.

Otra de las cuestiones que se ha evidenciado durante la pandemia también ha evidencia la brecha digital existente entre diferentes grupos sociales, ya sea por cuestiones de renta, clase social o edad. Ya sabemos que el advenimiento de nuevas tecnologías ha cambiado muchos los comportamientos sociales, la manera de enseñar y aprender e incluso de relacionarse y mantener el contacto con nuestros familiares y seres queridos. Es conveniente que nuestros mayores se adapten lo más rápido posible a estos cambios. Para ello sería necesario que los poderes públicos tomen las medidas pertinentes de manera inmediata para hacer posible esta adaptación, ofreciendo formación, facilidades económicas o la adquisición de los materiales digitales o informáticos pertinentes.

Nuestros mayores tienen el derecho a participar de las grandes decisiones y dilemas de nuestro tiempo y que se plantean todas las sociedades modernas: la muerte digna, los cuidados de larga duración, la gestión de la dependencia, la brecha digital, el modelo residencial público y privado... Todo aquello pueda afectarles directa o indirectamente. Tenemos la obligación a informarles, conocer su opinión y ellos el derecho pleno a expresarla. 
AGULLÓ, M. S.; AGULLÓ, E. y RODRÍGUEZ, J. (2002): "Voluntariado de mayores: ejemplo de envejecimiento participativo y satisfactorio", Revista Interuniversitaria de Formación del Profesorado, 45, pp. 107128.

AMEZCUA, T. y ABERICH,T. (2020): “Personas mayores: motor de movimientos sociales posindustriales. La marea de pensionistas y jubilados en España y el movimiento Stuttgart 21 en Alemania", Encrucijadas: Revista Crítica de Ciencias Sociales, 19, pp.1-22.

LAYALON, L.; CHASTEEN, A.; DIEHL, M.; LEVY, B. R.; NEUPERT, S. D.; ROTHERMUND, K.; TESCH-RÖMER, C. y WAHL, H. W. (2020): “Aging in Times of the COVID-19 Pandemic: Avoiding Ageism and Fostering Intergenerational Solidarity", The Journals of Gerontology Series B Psychological Sciences and Social Sciences, 76 (2), e49-e52. Doi: 10.1093/geronb/gbaa051. PMID: 32296840; PMCID: PMC7184502.

BARTH, J.; SCHNEIDER, S. y VON KANEL, R. (2010): “Lack of social support in the etiology and the prognosis of coronary heart disease: A systematic review and meta-analysis", Psychosomatic Medicine, 72, pp. 229-238.

BJØRKØY, A. M. B. (2017): “Ageing and dignity”, en P. Andersen (ed.), Literature and honour. Oslo, Universitetsforlaget, pp. 249-274.

BOWLIN, A. y GRUNDY, E. (2009): “Differentials in mortality up to 20 years after baseline interview among older people in East London and Essex", Age and Ageing, 38, pp. 51-55.

BROOK, J. y JACKSON, D. (2020): “Older people and COVID-19: Isolation, risk and ageism", Journal of Clinical Nursering, 29, pp. 2044-2046.

CALERO, M. D.; GARCIA, T. M.; GÁLVEZ, M.; TORAL, I. y BAILÓN, R. (1996): "Valoración de un programa de autoayuda entre ancianos: perfil de los voluntarios", Intervención Psicosocial, 5(13), pp. 85-95.

CAMPAIGN TO END LONELINESS (2011): Safeguarding the Convoy: A call to action from the Campaign to End Loneliness, https://www.campaigntoendloneliness.org/wp-content/uploads/Safeguarding-theConvoy.-A-call-to-action-from-the-Campaign-to-End-Loneliness. pdf.

CÁRITAS ESPAÑOLA (2009): Voluntariado y personas mayores. Material formativo. Madrid: Cáritas Española Editores.

CHRISTENSEN, K.; DOBLHAMMER, G.; RAU, R. y VAUPEL, J. W. (2009): "Ageing population: in the challenged ahead", The Lancet, 374, pp. 1198-1208.

COHEN-MANSFIELD, J.; HAZAN, H.; LERMAN, Y. y SHALOM, V. (2016): "Correlates and predictors of loneliness in older-adults: A review of quantitative results informed by qualitative insights", International Psychogeriatrics, 28 (4), pp. 557-576.

DEL BARRIO, E. (2007): Uso del tiempo entre las personas mayores. Boletín sobre el envejecimiento, IMSERSO, 27.

EDUCO (2015): Informe Crisis y efecto dominó ¿Quedan piezas por caer? El bienestar infantil, abuelas y abuelos en la brecha. Barcelona, Educo.

FAMILY MATTERS INSTITUTE (2009): Do grandparents' matter? The impact of grandparenting on the wellbeing of children. Bedford, Family Matters Institute.

FERNÁNDEZ-BALLESTEROS, R. (2003): "Una perspectiva psico-social: Promoción del envejecimiento activo", en Longevidad y vida saludable. Madrid, AECES.

FINGERMAN, K. L.; y TREVINO, K. (2020): “Don't lump seniors together on coronavirus. Older people aren't all the same", USA Today, https:// eu.usatoday.com/story/opinion/2020/04/07/coronavirus-seniorslead-diverse-lives-death-rate-varies-column/2954897001/.

HATTO-YEO, A. (2007): Ageing and social policy: a report for volunteering in the third age. Oxford, Volunteering in the Third Age. The Beth Johnson Foundation
HAVIGHURST, R. J y ALBRETCH, R. (1953): Older people. Nueva York, Longmans Gree.

HAVIGHURST, R. J.; NEUGARTEN, B. y TOBIN, S. S. (1963): “Disengagement, personality and life satisfaction in the later years", en P. Hansen (ed.), Age with a future. Copenhagen, Munksgoard, pp. 419-425.

HERNANDIZ, S. P.; RUIZ, M. T.; MARTÍ, M. J. y MOLINA, M. B. (2019): “Participación social y satisfacción vital: diferencias entre mujeres y hombres mayores", Revista de psicología de la salud, 7 (1), pp. 202-234.

HOLT-LUNSTAD, J.; SMITH, T. B. y LAYTON, J. B. (2010): "Social relationships and mortality risk: A meta-analytic review", PLoS Med, 7(7).

IMSERSO (2008): La participación social de las Personas mayores. Madrid, IMSERSO.

IMSERSO (2011:) Libro Blanco sobre Envejecimiento Activo. Madrid, IMSERSO.

INE (2017): Encuesta continua de hogares. Madrid, INE.

INE (2018a): Estadística del padrón Continuo. Datos provisionales a 1 de enero de 2018. Madrid, INE.

INE (2018b): Encuesta de Población Activa, tercer trimestre, 2018. Madrid, INE.

INE (2021): Avance del Padrón continuo. Resultados Provisionales a 1 de julio de 2020. Madrid, INE.

KELLY, J. (1993): Activity and aging. Staying involved in later life. Newbury Park, CA, Sage.

MCADAM, D.; J. MCCARTHY y M. ZALD. (1999): Movimientos sociales, perspectivas comparadas: oportunidades políticas, estructuras de movilización y marcos interpretativos culturales. Madrid, AKAL.

MEDINA TORNERO, M. E. (2005): Voluntariado y mayores. Murcia, Plataforma del Voluntariado de la Región de Murcia.

MEDINA, E. (2015): Satisfacción e intención de permanencia de personas mayores voluntarias. Uno modelo explicativo. (Tesis inédita), Universidad de Murcia. Facultad de Trabajo Social.

MEDINA, E. MARCOS-MATÁS, G. y MEDINA, M. E. (2016): “Older volunteers' intention to remain in service in nonprofit organisation", Psicothema, 28 (3), pp. 272-277.

MINISTRY OF SOCIAL DEVELOPMENT (2001): New Zealand positive ageing strategy. Wellington, New Zealand, Ministry of Social Development.

MIRANDA, A. G.; HERNANDO, L. O.; MARTÍNEZ, A. P.; MORALES, M. M. y OBERGOZO, A. (2006): Envejecimiento activo, envejecimiento en positivo. Logroño, Universidad La Rioja

MORGADO, G. y ROMÁN, M. (2011): “La familia como contexto del desarrollo infantil", en V. Muñoz y cols. (eds.), Manual de psicología del desarrollo aplicada a la educación. Madrid, Pirámide, pp. 37-60.

NAVARRIO CARRIÓ, M. (2000): Políticas sociales para los mayores en la Comunidad Autónoma de Murcia en Políticas sociales para las personas mayores en el próximo siglo. Murcia, Caja de Ahorros del Mediterráneo-Universidad de Murcia, pp. 159-173

OBRA SOCIAL FUNDACIÓN “LA CAIXA”. (2020): La soledad en las personas mayores: prevalencia, características y estrategias de afrontamiento. Barcelona, Obra Social La Caixa.

PINAZO-HERNANDIS, S. (2020): "Impacto psicosocial de la COVID-19 en las personas mayores: problemas y retos", Revista Española de Geriatría y Gerontología, 55 (5), pp. 249-252.

POSNER, R. M. (1995): Aging and Old Age. Chicago, University of Chicago Press

RIESCO VÁZQUEZ, E. (2014): La vejez y la política. Participación y potencial político de los mayores en España. Del voto cautivo al poder gris. (Tesis doctoral), Universidad de Salamanca. Salamanca, España.

RUBIO, A. (2004): "Perspectivas teóricas en el estudio de los movimientos sociales", Circunstancia: revista de ciencias sociales del Instituto Universitario de Investigación Ortega y Gasset, 3, pp. 4-12.

SANTINI, Z.; JOSE, P.; CORNWELL, E.; KOYANAGI, A.; NIELSEN, L.; HINRICHSEN, C. y KOUSHEDE, V. (2020): "Social disconnectedness, perceived isolation, and symptoms of depression and anxiety among older Americans (NSHAP): A longitudinal mediation analysis", Lancet Public Health, 5, pp. 62-70. 
SERRAT FERNÁNDEZ, R. (2016): La participación de las personas mayores en organizaciones políticas: modelos explicativos entrados en el individuo. (Tesis doctoral), Universidad de Barcelona. Barcelona, España.

SHANKAR, A.; MCMUNN, A.; DEMAKAKOS, P.; HAMER, M. y STEPTOE, A. (2017): "Social isolation and loneliness: Prospective associations with functional status in older adults", Health Psychology, 36 (2), pp. 179-187.

STEPTOE, A.; SHANKAR, A.; DEMAKAKOS, P. y WARDLE, J. (2013): “Social isolation, loneliness, and all-cause mortality in older men and women", Proceedings of the National Academy of Sciences of the United States of America, 110, pp. 5797-5801.

TANSKANEN, J. y ANTTILA, T. A. (2016): "Prospective study of social isolation, loneliness, and mortality in Finland", American Journal of Public Health, 106, pp. 2042-2048.

TRIADÓ, M. C. (2003): Envejecer en Entornos Rurales. Madrid, IMSERSOEstudios I+D+l, 19.

UPD (2013): Voluntariado Social UDP: Tal como somos. Madrid, Unión Democrática de Pensionistas y Jubilados de España (UPD).

VALTORTA, N. y HANRATTY, B. (2012): “Loneliness, isolation and the health of older adults: Do we need a new research agenda?", Journal of the Royal Society of Medicine, 105 (12), pp. 518-522.

VÁZQUEZ DE PRADA, M. (2005): “Para una historia de la familia española en el siglo XX", en Memorias y Civilización. Pamplona, Universidad de Navarra, pp. 115-170.

VEGA, J. L. V.; DELGADO, J. B. y MARTÍNEZ, M. B. B. (2002): “Niveles de actividad y participación social en las personas mayores de 60 años", Revista Interuniversitaria de Formación del profesorado, 45, pp. 33-35.

VICTOR, C. R. y BOWLING, A. (2012): "A longitudinal analysis of loneliness among older people in Great Britain", The Journal of Psychology, 146 (3), 313-331.

VICTOR, C. R.; SCAMBLER, S. J.; BOWLING, A. y BOND, J. (2005): "The prevalence of, and risk factors for, loneliness in later life: A survey of older people in Great Britain", Ageing and Society, 25 (6), pp. 357-375. 